\title{
Butorphanol alleviates lipopolysaccharide-induced inflammation and apoptosis of cardiomyocytes via activation of the $\kappa-$-pioid receptor
}

\author{
WEIQING TANG ${ }^{1}$, LIU LUO ${ }^{2}$, BAOJI HU ${ }^{1}$ and MINGZHI ZHENG ${ }^{2}$ \\ ${ }^{1}$ Department of Anesthesiology, Shanghai Pudong Hospital, Fudan University Pudong Medical Center, \\ Shanghai 201399; ${ }^{2}$ Department of Anesthesiology, The Affiliated Zhuzhou Hospital of Xiangya School of \\ Medicine, Central South University, Zhuzhou Central Hospital, Zhuzhou, Hunan 412000, P.R. China
}

Received November 9, 2020; Accepted April 16, 2021

DOI: 10.3892/etm.2021.10683

\begin{abstract}
Sepsis-induced myocardial dysfunction is a leading cause of the high mortality rates associated with sepsis. The aim of the present study was to investigate the effect of butorphanol on sepsis-induced cardiomyocyte dysfunction. Lipopolysaccharide (LPS) was used to induce H9C2 cardiomyocytes to establish an in vitro sepsis model. The effect of butorphanol on the viability of LPS-induced H9C2 cells was analyzed using a Cell Counting Kit- 8 assay. The levels of tumor necrosis factor- $\alpha$, interleukin (IL)- $1 \beta$ and IL- 6 were detected using ELISA. Western blotting was used to analyze the expression levels of inflammation-and apoptosis-related proteins. Cell apoptosis was measured using a TUNEL assay. The expression levels of $\kappa$-opioid receptor (KOR) were analyzed using reverse transcription-quantitative PCR analysis and western blotting. Following LPS induction, the levels of inflammatory cytokines and proapoptotic proteins were found to be upregulated in H9C2 cells, while butorphanol treatment downregulated these levels. The expression levels of KOR were also upregulated following butorphanol treatment in LPS-induced H9C2 cells. Addition of the KOR inhibitor, nor-binaltorphimine, alleviated the inhibitory effects of butorphanol on inflammation and apoptosis in LPS-induced H9C2 cells. In conclusion, the findings of the present study provided evidence indicating that butorphanol may alleviate LPS-induced inflammation and apoptosis in cardiomyocytes by upregulating KOR expression, which may provide a novel
\end{abstract}

Correspondence to: Dr Mingzhi Zheng, Department of Anesthesiology, The Affiliated Zhuzhou Hospital of Xiangya School of Medicine, Central South University, Zhuzhou Central Hospital, 116 Changjiang South Road, Tianyuan, Zhuzhou, Hunan 412000, P.R. China

E-mail: mingzhizmz@163.com

Key words: butorphanol, lipopolysaccharide, к-opioid receptor, sepsis insight into the potential therapeutic effects of butorphanol and its underlying mechanism of action.

\section{Introduction}

Sepsis is a systemic inflammatory response triggered by infection, which is often accompanied by fever, leukopenia and multiorgan failure $(1,2)$. Although it is common, sepsis is associated with high mortality rates and often requires patients to be admitted into intensive care units $(1,3)$. The incidence rate of sepsis is currently 66-300 per 100,000 individuals in developed countries, and continues to increase $(4,5)$. Results of a previous study indicated that almost half of patients with sepsis have clinical manifestations associated with myocardial dysfunction (6). Sepsis-induced myocardial dysfunction (SIMD) is a leading cause of the high mortality rates observed in sepsis (7).

Butorphanol, a US Food and Drug Administration-approved analgesic agent, is a potent $\kappa$-opioid receptor (KOR) agonist and $\mu$-opioid receptor (MOR) antagonist, which binds to KORs and modifies their expression (8). It was previously reported that butorphanol was successfully used to treat patients with moderate or severe pruritus (9-11). Comparative studies demonstrated that butorphanol was $\sim 7$-fold more effective compared with morphine sulphate, a full MOR agonist $(12,13)$. A previous meta-analysis also reported that butorphanol exerted significant therapeutic effects by reducing the incidence and severity of opioid-induced cough (14). In addition, butorphanol was found to be an optimal drug for prescribing to patients in labor with chronic hypertension or preeclampsia, as it did not elevate blood pressure (15). Previous studies have also shown that butorphanol alleviated myocardial ischemia/reperfusion-induced injury in rats $(16,17)$. Recent evidence has suggested a protective role for butorphanol against sepsis-induced cerebral injury. The aim of the present study was to determine the potential role of butorphanol in the inflammation and apoptosis mediated by KOR in lipopolysaccharide (LPS)-induced cardiomyocytes (18).

LPS is a crucial component of the outer membrane of Gram-negative bacteria, which plays a significant role in Gram-negative bacterial infection and induces myocardial 
damage (19). The present study used LPS to induce inflammation and apoptosis in cardiomyocytes in order to investigate the effects of butorphanol in an in vitro study of SIMD.

\section{Materials and methods}

Cell culture and experimental grouping. H9C2 cardiomyocytes were purchased from The Cell Bank of Type Culture Collection of the Chinese Academy of Sciences. Cells were cultured in DMEM (Gibco; Thermo Fisher Scientific, Inc.) supplemented with $100 \mathrm{~g} / \mathrm{ml}$ streptomycin, $100 \mathrm{~g} / \mathrm{ml}$ penicillin and 10\% FBS (Gibco; Thermo Fisher Scientific, Inc.), and maintained in a humidified atmosphere of $5 \% \mathrm{CO}_{2}$ at $37^{\circ} \mathrm{C}$. $\mathrm{H} 9 \mathrm{C} 2$ cells were treated with $1.25,2.5$ or $5 \mu \mathrm{M}$ butorphanol $(1 \mathrm{mg} / \mathrm{mL}$, Butorphanol (Jiangsu Hengrui Medicine Co. Ltd.) for $24 \mathrm{~h}$ at $37^{\circ} \mathrm{C}$ for further analysis. Cells were divided into the following four groups: i) H9C2 group, in which cells were cultured in basic culture medium; ii) LPS group, in which cells were treated with $10 \mu \mathrm{g} / \mathrm{ml}$ LPS from Escherichia coli O111:B4 (Sigma-Aldrich; Merck KGaA) for $24 \mathrm{~h}$; iii) LPS $+1.25,2.5$ or $5 \mu \mathrm{M}$ butorphanol group, in which cells were pretreated with $1.25,2.5$ or $5 \mu \mathrm{M}$ butorphanol for $2 \mathrm{~h}$ prior to treatment with $10 \mathrm{~g} / \mathrm{ml}$ LPS for $24 \mathrm{~h}$ (a preliminary experiment was conducted, which determined that $2 \mathrm{~h}$ was the appropriate pre-conditioning time of butorphanol); and iv) LPS $+5 \mu \mathrm{M}$ butorphanol + KOR antagonist nor-binaltorphimine (Nor-BNI; Abcam) group, in which cells were pretreated with $10 \mu \mathrm{mol} / 1$ Nor-BNI (20) for $30 \mathrm{~min}$ prior to the treatment described for the LPS $+5 \mu \mathrm{M}$ butorphanol group. After the experimental treatment, the cells were used for further analysis.

Cell Counting Kit (CCK)-8 assay. H9C2 cells were digested with Trypsin (cat. no. R001100; Gibco; Thermo Fisher Scientific, Inc.) and seeded into 96-well plates at a density of $3 \times 10^{5}$ cells/well. Following treatment with $10 \mu \mathrm{g} / \mathrm{ml}$ LPS and $1.25,2.5$ or $5 \mu \mathrm{M}$ butorphanol for $24 \mathrm{~h}$, the cells were incubated with $10 \mu$ l CCK- 8 reagent (MedChemExpress) for $4 \mathrm{~h}$ at $37^{\circ} \mathrm{C}$. The absorbance of each well was measured at a wavelength of $450 \mathrm{~nm}$ using a microplate reader. The cell viability was calculated using the formula: Cell viability $(\%)=\left[\mathrm{A}_{\text {drug }}-\mathrm{A}_{\text {blank }} / \mathrm{A}_{\text {odrug }}-\mathrm{A}_{\text {blank }}\right] \times 100 . \mathrm{A}_{\text {drug }}$, absorbance of wells containing cells, CCK8 solution and drug solution; $\mathrm{A}_{\text {blank }}$, absorbance of wells containing medium and CCK-8 solution, without cells; $\mathrm{A}_{0 \mathrm{drug}}$, absorbance of wells containing cells and CCK-8 solution, but no drug solution.

ELISA. After H9C2 cells ( $1 \times 10^{6}$ cells) were seeded into 6-well plates and treated as aforementioned, they were harvested and centrifuged at $3,000 \mathrm{xg}$ for $5 \mathrm{~min}$. The levels of tumor necrosis factor (TNF)- $\alpha$ (cat. no. ab181421), IL-1 $\beta$ (cat. no. ab214025) and IL-6 (cat. no. ab178013) in the H9C2 cell supernatant were detected using the respective commercial ELISA kits (Abcam) according to the manufacturer's protocols.

Western blotting. Total protein was extracted from $\mathrm{H} 9 \mathrm{C} 2$ cells using RIPA lysis buffer (Beyotime Institute of Biotechnology). Protein concentration was quantified using a BCA assay kit (cat. no. ab102536; Abcam) according to the manufacturer's protocol. A total of $50 \mu \mathrm{g}$ protein was loaded into each lane, separated by SDS-PAGE on $12 \%$ gels and subsequently transferred onto PVDF membranes (EMD Millipore), which were blocked with 5\% skimmed milk at room temperature for $1 \mathrm{~h}$. The membranes were then incubated with the following primary antibodies: Anti-phosphorylated (p)-P65 (cat. no. ab183559; 1:1,000; Abcam), anti-P65 (cat. no. ab32536; 1:1,000; Abcam), anti-cyclooxygenase 2 (cat. no. ab179800; 1:1,000; Abcam), anti-inducible nitric oxide synthase (cat. no. ab178945; 1:1,000; Abcam), anti-Bax (cat. no. ab32503; 1:1,000; Abcam), anti-cleaved caspase 3 (cat. no. ab32042; 1:1,000; Abcam), anti-cleaved poly(ADP)-ribose polymerase (PARP1; cat. no. ab32064; 1:1,000; Abcam), anti-Bcl-2 (cat. no. ab32124; 1:1,000; Abcam) and anti-GAPDH (cat. no. ab9485; 1:1,000; Abcam). Following incubation with the primary antibody at $4^{\circ} \mathrm{C}$ overnight, the membranes were incubated with the corresponding HRP-conjugated secondary antibody: i) rabbit anti-mouse secondary (cat. no. SA5-10317; 1:10,000; Invitrogen; Thermo Fisher Scientific, Inc.) and ii) goat anti-rabbit (cat. no. A16104SAMPLE; 1:10,000; Invitrogen; Thermo Fisher Scientific, Inc.) at room temperature for $2 \mathrm{~h}$. Protein bands were visualized using enhanced chemiluminescence reagent (Pierce; Thermo Fisher Scientific, Inc.) and analyzed using Image J software version 1.46r (National Institutes of Health). Protein expression levels were normalized to GAPDH expression levels.

TUNEL assay. $\mathrm{H} 9 \mathrm{C} 2$ cells were harvested, washed with PBS three times and fixed with $4 \%$ paraformaldehyde at room temperature for $20 \mathrm{~min}$. The TUNEL assay was then conducted using an In Situ Cell Death Detection Kit, Fluorescein (Roche Diagnostics) according to the manufacturer's protocol. The nuclei were counterstained with DAPI $(0.1 \mu \mathrm{g} / \mathrm{ml})$ at room temperature in the dark for $5 \mathrm{~min}$. TUNEL-positive cells were visualized using a fluorescence microscope (Olympus Corporation) and the percentage of apoptotic cells was calculated in five randomly selected fields of view. The apoptotic rate was analyzed using Image J $1.52 \mathrm{r}$ software and calculated as follows: Apoptosis rate $(\%)=($ Green fluorescence intensity/blue fluorescence intensity) x 100.

Reverse transcription-quantitative PCR (RT-qPCR). Total RNA was extracted from $\mathrm{H} 9 \mathrm{C} 2$ cells treated with LPS using TRIzol $^{\circledR}$ reagent (Invitrogen; Thermo Fisher Scientific, Inc.). RNA concentration was measured using a spectrophotometer. Total RNA was reverse-transcribed into cDNA using a Reverse Transcription kit (Beijing TransGen Biotech Co., Ltd.) according to the manufacturer's protocol. qPCR was performed using TransScript II Green Two-Step qRT-PCR SuperMix (cat. no. AQ301-01; TransGen Biotech Co., Ltd.) on a QuantStudio 6 Flex Real-Time PCR system (Thermo Fisher Scientific, Inc.). The thermocycling conditions were as follows: Initial denaturation at $94^{\circ} \mathrm{C}$ for $30 \mathrm{sec} ; 40$ cycles at $94^{\circ} \mathrm{C}$ for $5 \mathrm{sec}$, annealing at $55^{\circ} \mathrm{C}$ for $15 \mathrm{sec}$ and extension at $72^{\circ} \mathrm{C}$ for $10 \mathrm{sec}$. The relative mRNA expression levels of KOR were quantified using the $2^{-\triangle \Delta \mathrm{Cq}}$ method (21) and normalized to GAPDH. The primers used were as follows: KOR forward, 5'-GATGTG GATGTCATTGAATGCTCCTTGCAG-3' and reverse, 5'-GC TGTGCTGTGGGAGGTGCTGCCTAGAGCC-3'. GAPDH forward, 5'-AGGCCGGTGCTGAGTATGTC-3' and reverse, 5'-TGCCTGCTTCACCACCTTCT-3'. 

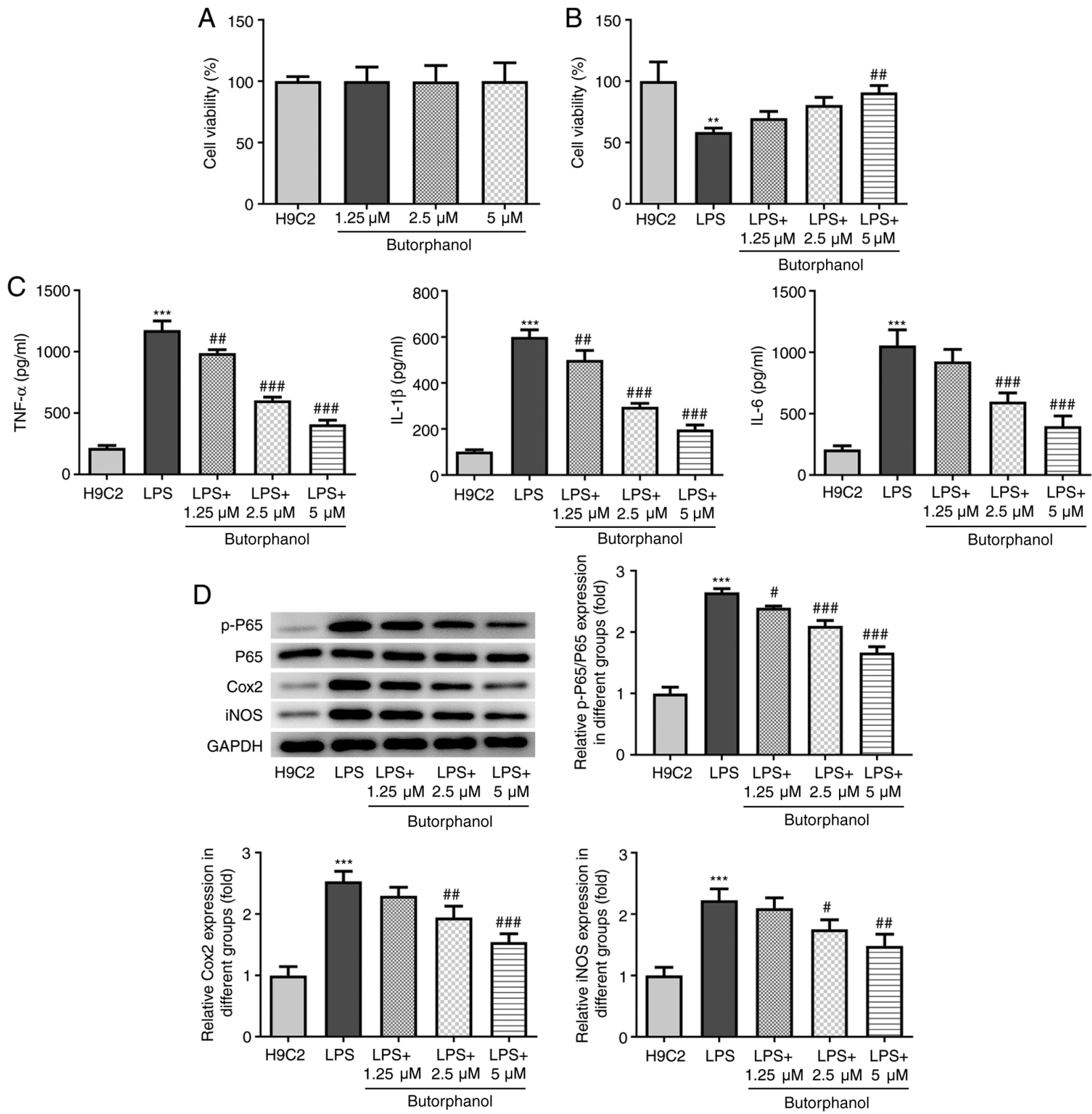

Figure 1. Butorphanol suppresses the release of inflammatory factors in LPS-induced cardiomyocytes. (A) LPS-induced H9C2 cell viability was detected by the Cell Counting Kit- 8 assay. (B) Cell viability was analyzed after pretreatment of H9C 2 cells with butorphanol at $1.25,2.5$ and $5 \mu \mathrm{M}$ for $2 \mathrm{~h}$ followed by LPS stimulation for $24 \mathrm{~h} .{ }^{* *} \mathrm{P}<0.01 \mathrm{vs}$. H9C2 group; ${ }^{\# \#} \mathrm{P}<0.01$ vs. LPS group. (C) Inflammatory factor levels in LPS-induced H9C2 cells after butorphanol treatment at $1.25,2.5$ and $5 \mu \mathrm{M}$ were tested by ELISA. ${ }^{* * *} \mathrm{P}<0.001$ vs. H9C2 group; ${ }^{\# \#} \mathrm{P}<0.01,{ }^{\# \# \#} \mathrm{P}<0.001$ vs. LPS group. (D) Inflammation-related protein expression in LPS-induced H9C2 cells was measured by western blotting. ${ }^{* * *} \mathrm{P}<0.001$ vs. $\mathrm{H} 9 \mathrm{C} 2$ group; ${ }^{~} \mathrm{P}<0.05,{ }^{\# \#} \mathrm{P}<0.01$ and ${ }^{\# \# \#} \mathrm{P}<0.001$ vs. LPS group. All statistical analyses were performed using one-way ANOVA followed by Tukey's test. LPS, lipopolysaccharide; COX2, cyclooxygenase 2; iNOS, inducible nitric oxide synthase; p-, phosphorylated.

Statistical analysis. Experiments were repeated at least three times and data are expressed as the mean $\pm \mathrm{SD}$. Statistical analysis was performed using GraphPad Prism software, version 7.0 (GraphPad Software, Inc.). Statistical differences among different groups were performed using one-way ANOVA followed by Tukey's post hoc test for multiple comparisons. $\mathrm{P}<0.05$ was considered to indicate a statistically significant difference.

\section{Results}

Butorphanol suppresses the release of inflammatory factors in LPS-induced H9C2 cells. To determine the effects of butorphanol on normal cardiomyocytes, the viability of $\mathrm{H} 9 \mathrm{C} 2$ cells was detected through CCK-8 assay after butorphanol treatment at different concentrations. The results of the CCK- 8 assay revealed that $\mathrm{H} 9 \mathrm{C} 2$ cell viability was not altered, which indicated that butorphanol did not affect the viability of normal cardiomyocytes (Fig. 1A). H9C2 cells were subsequently induced with LPS to establish an in vitro sepsis cell model. As shown in Fig. 1B, LPS significantly reduced the viability of $\mathrm{H} 9 \mathrm{C} 2$ cells, whereas butorphanol treatment restored the reduced cell viability induced by LPS in a dose-dependent manner. Inflammation is an important process involved in sepsis-induced cardiac injury (22); thus, the levels of TNF- $\alpha$, 


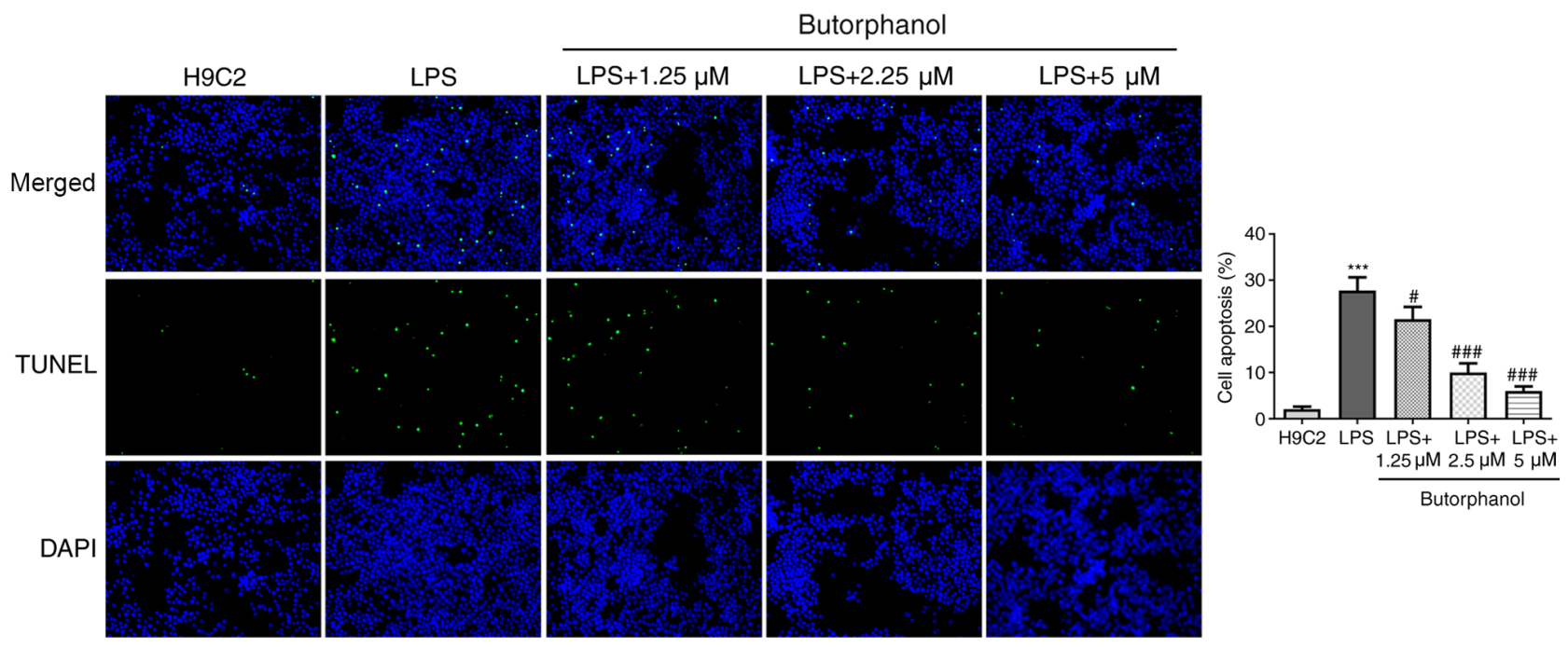

Figure 2. Butorphanol suppresses the apoptosis of LPS-induced cardiomyocytes. The effects of 1.25, 2.5 and $5 \mu \mathrm{M}$ butorphanol on the apoptosis of LPS-induced cardiomyocytes were measured by TUNEL assay. Magnification, $\mathrm{x} 200$. ${ }^{* * *} \mathrm{P}<0.001$ vs. H9C2 group; ${ }^{*} \mathrm{P}<0.05$ and ${ }^{\# \# \#} \mathrm{P}<0.001$ vs. LPS group. All statistical analyses were performed using one-way ANOVA followed by Tukey's test. LPS, lipopolysaccharide.

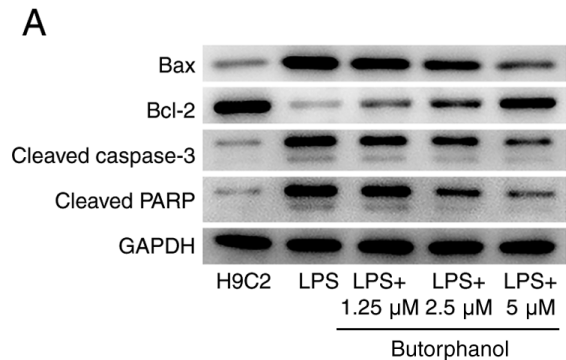

B
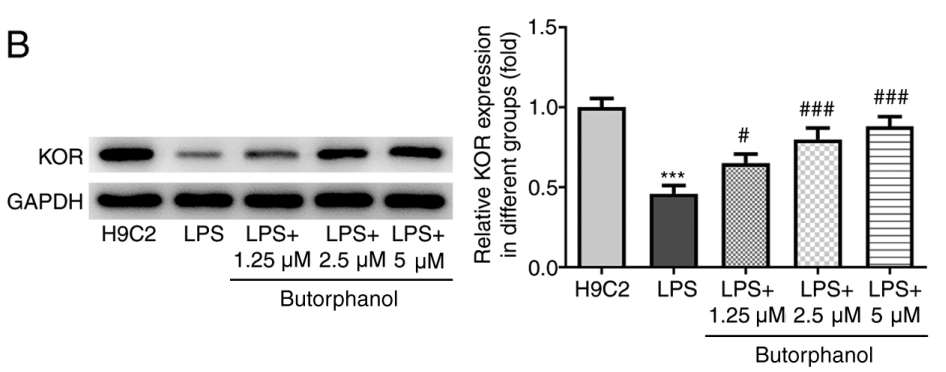
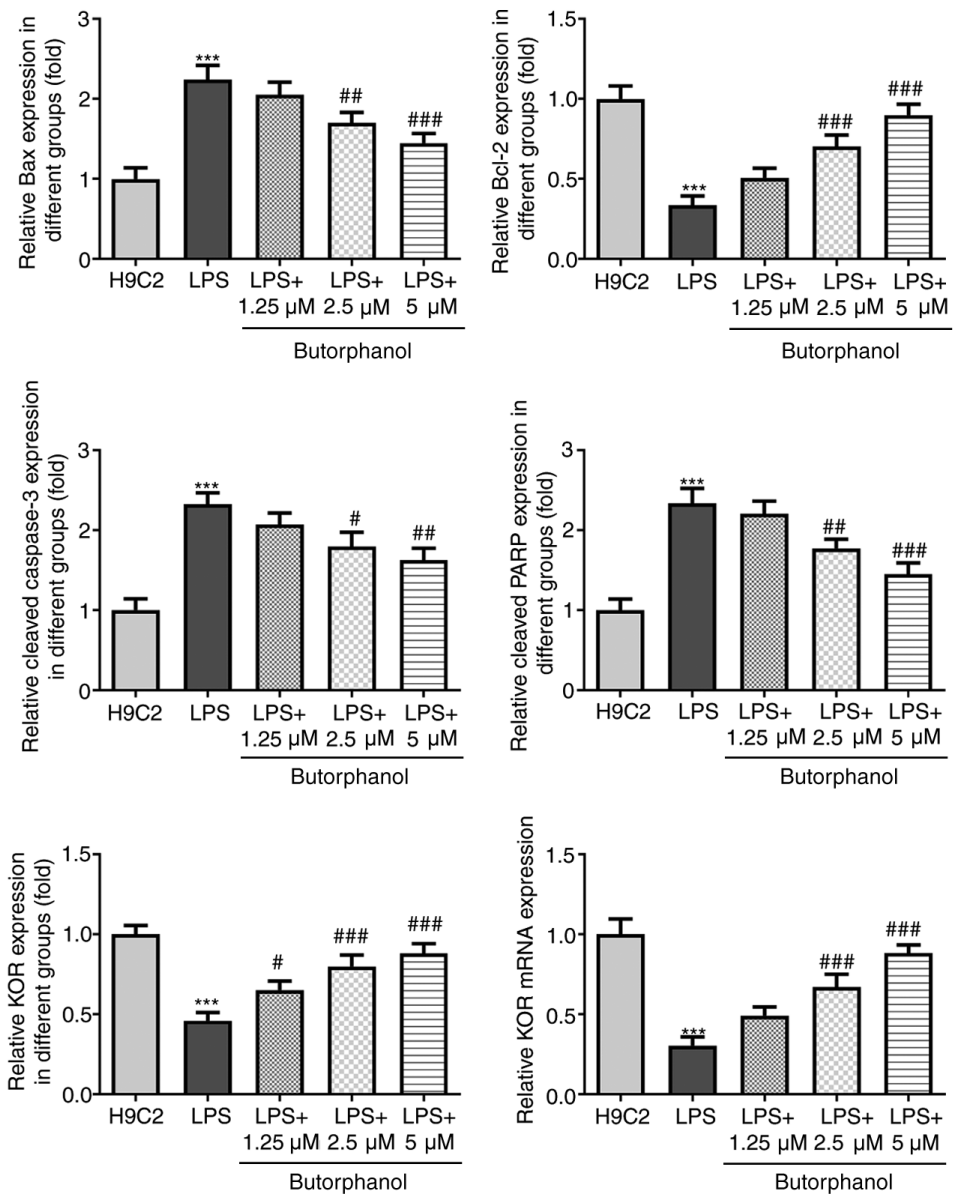

Figure 3. Butorphanol increases the expression of KOR in LPS-induced cardiomyocytes. (A) Protein expression levels of pro-apoptotic and anti-apoptotic proteins were measured by western blotting. ${ }^{* * *} \mathrm{P}<0.001$ vs. H9C2 group; ${ }^{\#} \mathrm{P}<0.05,{ }^{\# \#} \mathrm{P}<0.01$ and ${ }^{\# \#} \mathrm{P}<0.001$ vs. LPS group. (B) KOR expression was detected by western blotting and reverse transcription-quantitative PCR. ${ }^{* * *} \mathrm{P}<0.001$ vs. H9C2 group; ${ }^{\#} \mathrm{P}<0.05$, \#\# $\mathrm{P}<0.001$ vs. LPS group. All statistical analyses were performed using one-way ANOVA followed by Tukey's test. LPS, lipopolysaccharide; KOR, к-opioid receptor; PARP, poly(ADP)-ribose polymerase.

IL-1 $\beta$ and IL-6 were detected. As shown in Fig. 1C and D, LPS markedly elevated the levels of these inflammatory factors and upregulated the expression levels of inflammation-related proteins containing p-P65, iNOS and Cox2, whereas treatment with increasing doses of butorphanol gradually downregulated the levels of all inflammatory markers. 


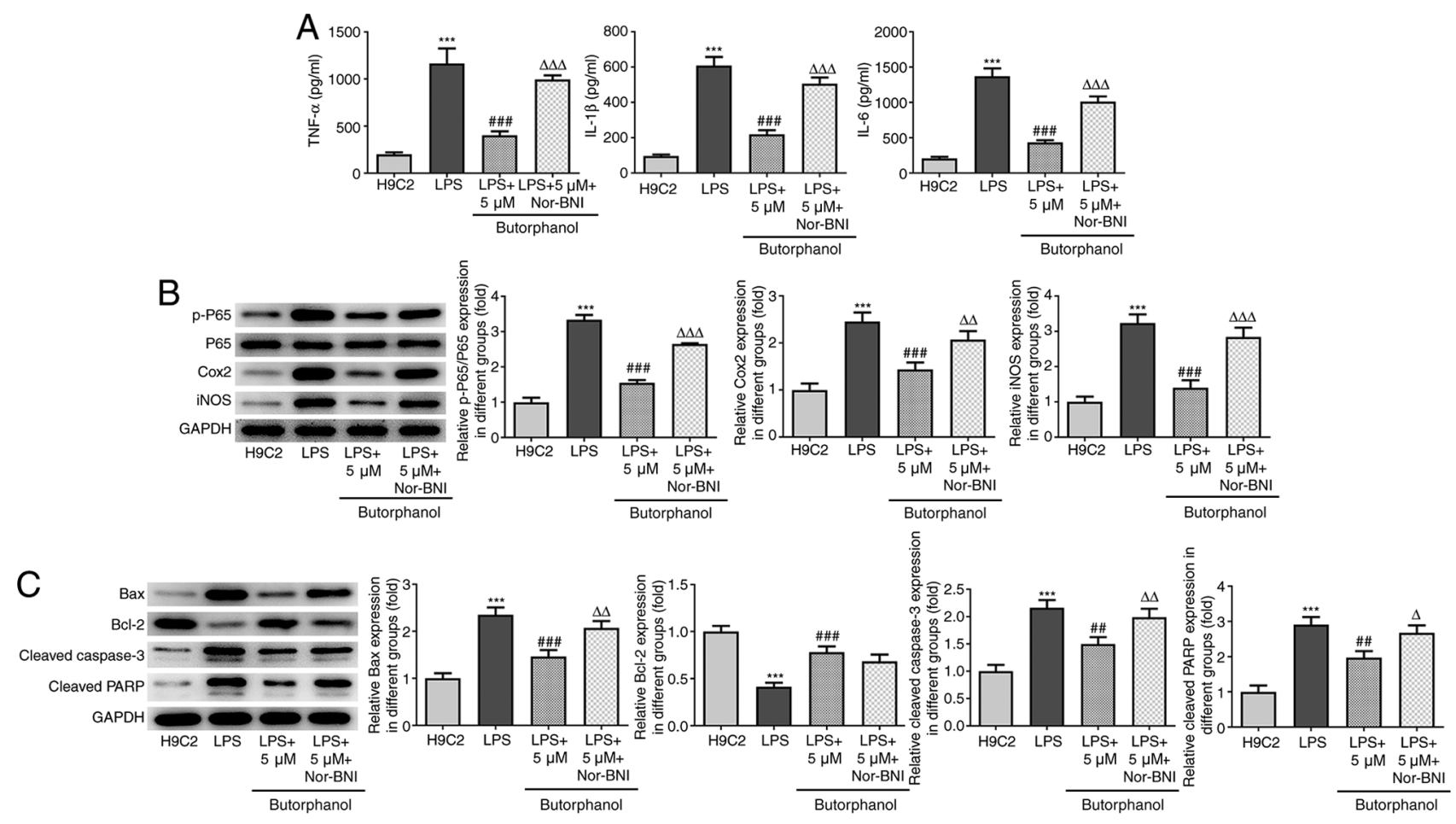

Figure 4. Butorphanol suppresses the release of inflammatory factors and apoptosis of LPS-induced cardiomyocytes by activating KOR. (A) The effect of Nor-BNI on the levels of inflammatory factors in LPS-induced H9C2 cells treated with $5 \mu \mathrm{M}$ butorphanol was evaluated by ELISA. (B) The effect of Nor-BNI on the protein expression levels of NF-кB P65, Cox 2 and iNOS was evaluated by western blotting. (C) The effect of Nor-BNI on the levels of apoptosis-related proteins was evaluated by western blotting. ${ }^{* * *} \mathrm{P}<0.001$ vs. H9C2 group; ${ }^{\# \#} \mathrm{P}<0.01,{ }^{\# \# \#} \mathrm{P}<0.001$ vs. LPS group; ${ }^{\Delta} \mathrm{P}<0.05,{ }^{\Delta \Delta} \mathrm{P}<0.01,{ }^{\Delta \Delta \Delta} \mathrm{P}<0.001$ vs. LPS $+5 \mu \mathrm{m}$ butorphanol group. All statistical analyses were performed using one-way ANOVA followed by Tukey's test. LPS, lipopolysaccharide; KOR, $\kappa$-opioid receptor; Nor-BNI, nor-binaltorphimine; Cox 2 cyclooxygenase 2; iNOS, inducible nitric oxide synthase; p-, phosphorylated; PARP, poly(ADP)-ribose polymerase.

Butorphanol suppresses the apoptosis of LPS-induced $\mathrm{H} 9 \mathrm{C} 2$ cells. Apoptosis is an important process in the occurrence of SIMD (23); therefore, the effect of butorphanol on the apoptosis of LPS-induced $\mathrm{H} 9 \mathrm{C} 2$ cells was investigated. As shown in Fig. 2, the apoptosis of $\mathrm{H} 9 \mathrm{C} 2$ cells was significantly elevated by LPS; however, butorphanol treatment reduced the apoptotic rate of $\mathrm{H} 9 \mathrm{C} 2$ cells. Concurrently, the expression levels of proapoptotic proteins (Bax, cleaved caspase 3 and cleaved PARP) were upregulated, whereas the expression levels of anti-apoptotic protein Bcl-2 were downregulated following LPS stimulation. These trends were all reversed following treatment of cells with butorphanol (Fig. 3A). These results suggested that butorphanol may suppress the apoptosis of LPS-induced H9C2 cells.

Butorphanol upregulates the expression of KOR in LPS-induced H9C2 cells. Butorphanol is known to act as a partial agonist of KOR in the G-protein activation signaling pathway (24). The results of western blot analysis revealed that LPS stimulation downregulated the expression levels of $\mathrm{KOR}$ in $\mathrm{H} 9 \mathrm{C} 2$ cells, whereas the expression levels of KOR were upregulated following butorphanol treatment (Fig. 3B). Of note, treatment with $5 \mu \mathrm{M}$ butorphanol upregulated the expression levels of KOR to the greatest extent. Additionally, 1.25 and $2.5 \mu \mathrm{m}$ butorphanol significantly increased the expression level of KOR compared with LPS group. These results suggested that butorphanol may upregulate the expression of KOR in LPS-induced H9C2 cells.
Butorphanol suppresses the release of inflammatory factors and the apoptosis of LPS-induced H9C2 cells by activating $K O R$. It was subsequently hypothesized that butorphanol may exert its anti-inflammatory and anti-apoptotic effects by activating KOR. The LPS-induced increases in secretory levels of inflammatory factors and expression levels of inflammation-related proteins were reduced following butorphanol treatment, whereas further addition of Nor-BNI, an inhibitor of KOR, reversed this trend (Fig. 4A and B). Western blot analysis also revealed that butorphanol treatment downregulated the expression levels of proapoptotic proteins; Bax, cleaved caspase 3 and cleaved PARP; however, these trends were subsequently reversed by the addition of Nor-BNI (Fig. 4C). There was no significant difference in the expression levels of the anti-apoptotic protein Bcl-2 between the butorphanol and Nor-BNI groups. Taken together, these findings indicated that butorphanol may suppress the release of inflammatory factors and the apoptosis of LPS-induced $\mathrm{H} 9 \mathrm{C} 2$ cells by upregulating KOR expression.

\section{Discussion}

Sepsis can cause multiorgan failure, and SIMD is the most complex and multifactorial type of organ failure (25). Myocardial dysfunction commonly occurs as a result of sepsis, affecting $64 \%$ of all patients with sepsis (25). However, to the best of our knowledge, the pathophysiology of SIMD has not been fully elucidated and SIMD requires further study. Butorphanol is widely used in the clinical setting. A 
previous study reported the beneficial therapeutic effects of butorphanol, such as improving pathology abnormalities and inflammation in a rat model of sepsis with brain injury (18). In addition, the transnasal application of butorphanol was able to alleviate pain in patients with acute migraines (26). However, current research mainly focuses on investigating the analgesic effects of butorphanol, whereas its effects on diseases such as SIMD remain poorly understood. Therefore, the present study aimed to investigate the effects of butorphanol on SIMD and elucidate the potential underlying mechanism.

Several previous studies investigating the underlying mechanisms of sepsis have confirmed that LPS $(10 \mathrm{mg} / \mathrm{kg})$, when injected intravenously into rats with septic shock, caused myocardial contraction and myocardial damage (27-29). Similarly, the present study used LPS to establish a cell model of sepsis, as SIMD is a major complication following the occurrence of septic shock (30). SIMD is a severe complication caused by inflammation and it has been suggested that the activation of inflammasomes may be involved in myocardial tissue damage (31). In the present study, the release of inflammatory cytokines was increased following LPS stimulation of H9C2 cells, whereas butorphanol suppressed the release of inflammatory factors. A previous study also reported that the levels of inflammatory cytokines were increased in sepsis-induced myocardial injury in rats (23). Apoptosis was found to play an important role in the occurrence of SIMD (23). Similar to its effects on inflammation, butorphanol treatment decreased the levels of apoptosis in LPS-induced $\mathrm{H} 9 \mathrm{C} 2$ cells.

As a member of the $\mathrm{G}$ protein-coupled receptor superfamily, KOR is the product of a single gene, KOR1, in species including pigs and humans (32-34). KOR internalization has been reported to be induced by butorphanol, which is a crucial step in the receptor signaling transduction cascade, which can bring about pharmacological changes that are associated with the efficacy and side effects of opioids (35). Previous studies have reported that the opioid receptor comprises the MOR, $\delta$-opioid receptor and KOR, and is not the product of a distinct gene; butorphanol shares a binding site with these three major opioid receptors, with an affinity ratio of 1:4:25, respectively $(36,37)$. KOR activation was reported to elicit anti-inflammatory and suppress pro-apoptotic effects (38). As regards the mechanism of regulation, it was previously revealed that KOR activation was involved in regulating the STAT3-OPA1 pathway to facilitate mitochondrial fusion and inhibit cell apoptosis (39). In the present study, butorphanol treatment upregulated the expression levels of KOR in LPS-induced $\mathrm{H} 9 \mathrm{C} 2$ cells, suggesting that KOR was activated by butorphanol. Following pretreatment of butorphanol-treated LPS-induced H9C2 cells with the KOR inhibitor Nor-BNI, the inhibitory effects of butorphanol were reversed, indicating that butorphanol may exert inhibitory effects over inflammation and apoptosis in LPS-induced $\mathrm{H} 9 \mathrm{C} 2$ cells by activating KOR. Further investigation is required to determine whether butorphanol exerts the same effects on inflammation and apoptosis in a KOR-dependent manner. Additionally, the lack of in vivo validation of the findings of the present study is a potential limitation that can be addressed in future studies.

In conclusion, the present study provided evidence to suggest that butorphanol may alleviate LPS-induced inflammation and apoptosis of cardiomyocytes by activating KOR, which may help researchers more comprehensively understand the therapeutic effects of butorphanol and its underlying mechanism of action.

\section{Acknowledgements}

Not applicable.

\section{Funding}

No funding was received.

\section{Availability of data and materials}

The datasets used and/or analyzed during the current study are available from the corresponding author on reasonable request.

\section{Authors' contributions}

WQT, LL, BJH and MZZ made substantial contributions to the conception and design of the study, performed the experiments, interpreted the data and drafted and revised the manuscript for important intellectual content. WQT and MZZ confirm the authenticity of all the raw data. All authors read and approved the final manuscript.

\section{Ethics approval and consent to participate}

Not applicable.

\section{Patient consent for publication}

Not applicable.

\section{Competing interests}

The authors declare that they have no competing interests.

\section{References}

1. Kaukonen KM, Bailey M, Pilcher D, Cooper DJ and Bellomo R: Systemic inflammatory response syndrome criteria in defining severe sepsis. N Engl J Med 372: 1629-1638, 2015.

2. Delano MJ and Ward PA: Sepsis-induced immune dysfunction: Can immune therapies reduce mortality? J Clin Invest 126: 23-31, 2016.

3. Gaieski DF,Edwards JM, Kallan MJ and Carr BG: Benchmarking the incidence and mortality of severe sepsis in the United States. Crit Care Med 41: 1167-1174, 2013.

4. Dombrovskiy VY, Martin AA, Sunderram J and Paz HL: Rapid increase in hospitalization and mortality rates for severe sepsis in the United States: A trend analysis from 1993 to 2003. Crit Care Med 35: 1244-1250, 2007.

5. Keeley A, Hine P and Nsutebu E: The recognition and management of sepsis and septic shock: A guide for non-intensivists. Postgrad Med J 93: 626-634, 2017.

6. Lv X and Wang H: Pathophysiology of sepsis-induced myocardial dysfunction. Mil Med Res 3: 30, 2016.

7. Yao Y, Sun F and Lei M: miR-25 inhibits sepsis-induced cardiomyocyte apoptosis by targetting PTEN. Biosci Rep 38: 38, 2018.

8. Hua J, Miao S, Shi M, Tu Q, Wang X, Liu S, Wang G and Gan J: Effect of butorphanol on etomidate-induced myoclonus: A systematic review and meta-analysis. Drug Des Devel Ther 13: 1213-1220, 2019.

9. Dawn AG and Yosipovitch G: Butorphanol for treatment of intractable pruritus. J Am Acad Dermatol 54: 527-531, 2006. 
10. Bailey AG, Valley RD, Freid EB and Calhoun P: Epidural morphine combined with epidural or intravenous butorphanol for postoperative analgesia in pediatric patients. Anesth Analg 79: 340-344, 1994

11. Gunter JB, McAuliffe J, Gregg T, Weidner N, Varughese AM and Sweeney DM: Continuous epidural butorphanol relieves pruritus associated with epidural morphine infusions in children. Paediatr Anaesth 10: 167-172, 2000.

12. Heel RC, Brogden RN, Speight TM and Avery GS: Butorphanol: A review of its pharmacological properties and therapeutic efficacy. Drugs 16: 473-505, 1978.

13. Hoskin PJ and Hanks GW: Opioid agonist-antagonist drugs in acute and chronic pain states. Drugs 41: 326-344, 1991

14. Zhang J, Miao S, Tu Q, Shi M, Zou L, Liu S and Wang G: Effect of butorphanol on opioid-induced cough: A meta-analysis of randomized controlled trials. Drug Des Devel Ther 12: 3263-3268, 2018.

15. Garrity K, Jang A and Wagner S: Butorphanol use in laboring patients with preeclampsia or chronic hypertension. Pregnancy Hypertens 6: 288-290, 2016.

16. Wang H, Wang JL, Ren HW, He WF and Sun M: Butorphanol protects on myocardial ischemia/reperfusion injury in rats through MAPK signaling pathway. Eur Rev Med Pharmaco Sci 23: 10541-10548, 2019.

17. Huang LH, Li J, Gu JP, Qu MX, Yu J and Wang ZY: Butorphanol attenuates myocardial ischemia reperfusion injury through inhibiting mitochondria-mediated apoptosis in mice. Eur Rev Med Pharmacol Sci 22: 1819-1824, 2018.

18. Meng J, Jiang SJ, Jiang D and Zhao Y: Butorphanol attenuates inflammation via targeting $\mathrm{NF}-\kappa \mathrm{B}$ in septic rats with brain injury. Eur Rev Med Pharmacol Sci 23 (Suppl 3): 161-170, 2019.

19. Baradaran Rahim V, Khammar MT, Rakhshandeh H, Samzadeh-Kermani A, Hosseini A and Askari VR: Crocin protects cardiomyocytes against LPS-Induced inflammation. Pharmacol Rep 71: 1228-1234, 2019

20. Cui Y, Feng N, Gu X, Fu F, Li J, Guo H, Liu Y, Zhang S, Li J, Wang Y, et al: $\kappa$-Opioid receptor stimulation reduces palmitate-induced apoptosis via Akt/eNOS signaling pathway. Lipids Health Dis 18: 52, 2019.

21. Kenneth JL and Thomas DS: Analysis of relative gene expression data using real-time quantitative PCR and the $2-\Delta \Delta C T$ method. Methods 25: 402-408, 2002.

22. Liang W, Li J, Bai C, Chen Y, Li Y, Huang G and Wang X: Interleukin-5 deletion promotes sepsis-induced M1 macrophage differentiation, deteriorates cardiac dysfunction, and exacerbates cardiac injury via the NF- $\mathrm{kB}$ p 65 pathway in mice. Biofactors 46 : 1006-1017, 2020.

23. Yang C, Xia W, Liu X, Lin J and Wu A: Role of TXNIP/NLRP3 in sepsis-induced myocardial dysfunction. Int J Mol Med 44: 417-426, 2019

24. Ji J, Lin W, Vrudhula A, Xi J, Yeliseev A, Grothusen JR, Bu W and Liu R: Molecular interaction between butorphanol and א-opioid receptor. Anesth Analg 131: 935-942, 2020.

25. Pulido JN, Afessa B, Masaki M, Yuasa T, Gillespie S, Herasevich V, Brown DR and Oh JK: Clinical spectrum, frequency, and significance of myocardial dysfunction in severe sepsis and septic shock. Mayo Clin Proc 87: 620-628, 2012.

26. Hoffert MJ, Couch JR, Diamond S, Elkind AH, Goldstein J, Kohlerman NJ III, Saper JR and Solomon S: Transnasal butorphanol in the treatment of acute migraine. Headache 35: 65-69, 1995.
27. Afulukwe IF, Cohen RI, Zeballos GA, Iqbal M and Scharf SM: Selective NOS inhibition restores myocardial contractility in endotoxemic rats; however, myocardial NO content does not correlate with myocardial dysfunction. Am J Respir Crit Care Med 162: 21-26, 2000

28. Cohen RI, Wilson D and Liu SF: Nitric oxide modifies the sarcoplasmic reticular calcium release channel in endotoxemia by both guanosine-3',5' (cyclic) phosphate-dependent and independent pathways. Crit Care Med 34: 173-181, 2006.

29. Chagnon F, Bentourkia M, Lecomte R, Lessard M and Lesur O: Endotoxin-induced heart dysfunction in rats: Assessment of myocardial perfusion and permeability and the role of fluid resuscitation. Crit Care Med 34: 127-133, 2006.

30. Walley KR: Sepsis-induced myocardial dysfunction. Curr Opin Crit Care 24: 292-299, 2018.

31. Su Q, Li L, Sun Y, Yang H, Ye Z and Zhao J: Effects of the TLR4/Myd88/NF- $\mathrm{kB}$ signaling pathway on NLRP3 inflammasome in coronary microembolization-induced myocardial injury. Cell Physiol Biochem 47: 1497-1508, 2018.

32. Commiskey S, Fan LW, Ho IK and Rockhold RW: Butorphanol: Effects of a prototypical agonist-antagonist analgesic on kappa-opioid receptors. J Pharmacol Sci 98: 109-116, 2005.

33. Xie GX, Meng F, Mansour A, Thompson RC, Hoversten MT, Goldstein A, Watson SJ and Akil H: Primary structure and functional expression of a guinea pig kappa opioid (dynorphin) receptor. Proc Natl Acad Sci USA 91: 3779-3783, 1994.

34. Yasuda K, Espinosa R III, Takeda J, Le Beau MM and Bell GI: Localization of the kappa opioid receptor gene to human chromosome band 8q11.2. Genomics 19: 596-597, 1994.

35. Xu J, Chen F, Wang S, Akins NS, Hossain MI, Zhou Y, Huang J, Ji J, Xi J, Lin W, et al: Kappa opioid receptors internalization is protective against oxygen-glucose deprivation through $\beta$-arrestin activation and Akt-mediated signaling pathway. Neurochem Int 137: 104748, 2020

36. Simonin F, Slowe S, Becker JA, Matthes HW, Filliol D, Chluba J, Kitchen I and Kieffer BL: Analysis of [3H]bremazocine binding in single and combinatorial opioid receptor knockout mice. Eur J Pharmacol 414: 189-195, 2001.

37. Chang KJ, Hazum E and Cuatrecasas P: Novel opiate binding sites selective for benzomorphan drugs. Proc Natl Acad Sci USA 78: 4141-4145, 1981 .

38. Chéret J, Gherardini J, Soeberdt M, Hundt JE, Abels C, Bertolini $\mathrm{M}$ and Paus R: Non-neuronal kappa-opioid receptor activation enhances epidermal keratinocyte proliferation, and modulates mast cell functions in human skin ex vivo. J Dermatol 47: 917-921, 2020

39. Wang K, Liu Z, Zhao M, Zhang F, Wang K, Feng N, Fu F, $\mathrm{Li} \mathrm{J}, \mathrm{Li}$ J, Liu Y, et al: $\kappa$-opioid receptor activation promotes mitochondrial fusion and enhances myocardial resistance to ischemia and reperfusion injury via STAT3-OPA1 pathway. Eur J Pharmacol 874: 172987, 2020.

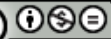

This work is licensed under a Creative Commons Attribution-NonCommercial-NoDerivatives 4.0 International (CC BY-NC-ND 4.0) License. 